\title{
Improving the repeatability of heterochromatic flicker photometry for measurement of macular pigment optical density
}

Olivia Howells, Frank Eperjesi, Hannah Bartlett

All authors: Ophthalmic Research Group, School of Life and Health Sciences, Aston University, Birmingham, B4 7ET, UK

Corresponding author: Frank Eperjesi: F.Eperjesi@aston.ac.uk; Tel. +44 121204 41141; Fax. +44 1212044048

No financial relationships with external organisations exist.

\section{ABSTRACT}

Background: Heterochromatic flicker photometry (HFP) is a psychophysical technique used to measure macular pigment optical density (MPOD). We used the MPS 9000 (MPS) HFP device. Our aim was to determine if the repeatability of the MPS could be improved to make it more suitable for monitoring MPOD over time.

Methods: Intra-session repeatability was assessed in 25 participants (aged 20-50). The resulting data was explored in detail, e.g., by examining the effect of removal and adjustment of data with less than optimal quality parameters. A protocol was developed for improved overall reliability, which was then tested in terms of inter-session repeatability in a separate group of 27 participants (aged 19-52).

Results: Removal and adjustment of data reduced the intra-session coefficient of repeatability (CR) by 0.04 , on average, and the mean individual standard deviation by 0.004 . Raw data observation offered further insight into ways of improving repeatability. The proposed protocol resulted in an inter-session CR of 0.08 .

Conclusions: Removal and adjustment of less than optimal data improved repeatability, and is therefore recommended. To further improve repeatability, in brief we propose that each patient 
perform each part of the test twice, and a third time where necessary (described in detail by the protocol). Doing so will make the MPS more useful in research and clinical settings.

Keywords: age-related macular degeneration; heterochromatic flicker photometry; macular pigment; macular pigment optical density 


\section{INTRODUCTION}

Macular pigment (MP) is the collective name for three carotenoids, lutein (L), zeaxanthin $(Z)$ and meso-zeaxanthin (MZ), which are found at high concentrations in the central macula, to the exclusion of all other carotenoids. ${ }^{1}$ It has received attention in recent years because of its possible links with the prevention of, and/or slowing the progression of, age-related macular degeneration (AMD). Lutein, $Z$ and $M Z$ are only available to the body by dietary intake of foods or supplements containing them, ${ }^{2,3}$ and it is possible to increase their concentration in the macula by regular consumption of foods and/or supplements rich in these carotenoids. ${ }^{4-6}$

Macular pigment is predominantly a blue light filter; its absorption spectrum peaks around 460 $\mathrm{nm} \cdot{ }^{7-10}$ It is well placed in the retina to reduce the amount of blue light reaching the photoreceptors; the higher the MP optical density (MPOD), the greater the amount of blue light filtering that will occur. ${ }^{11,12}$ It is also proposed that MP protects against oxidative stress, with evidence showing that $\mathrm{L}$ and $\mathrm{Z}$ have antioxidant properties. ${ }^{13,14}$ Since blue light and oxidative stress have been implicated in the pathogenesis of $A M D,{ }^{15,16}$ a high level of MP could reduce the risk for AMD, and there is an increasing amount of evidence to support this. ${ }^{11,17-20}$

Heterochromatic flicker photometry (HFP) is a psychophysical technique that makes use of the spectral absorption properties and retinal location of MP in order to measure MPOD. ${ }^{21}$ HFP determines MPOD by presenting a light stimulus of two alternating wavelengths at the fovea and at an eccentric retinal area (technically the parafovea or perifovea but often referred to interchangeably as either the parafoveal or peripheral measure), with the aim of achieving a perception of no or minimal flicker; this occurs when the luminances of the two wavelengths appear equal to the observer. ${ }^{22,23}$ The wavelengths are chosen so that one is a short-wavelength blue light that is maximally absorbed by MP, and the other is a longer-wavelength green to yellow light that is not absorbed by MP. In traditional HFP, the blue-green alternation frequency remains constant as the radiance of the blue light is increased or decreased until minimal flicker is reported. ${ }^{24-26}$ The procedure is carried out with the subject looking directly at the stimulus (i.e., the foveal measure), and then with the subject looking away from the stimulus so that the eccentric 
measure (where MP is assumed to be negligible) can be made. ${ }^{21}$ The presence of MP in the fovea (with subsequent absorption of some of the blue light before it reaches the photoreceptors) means that a greater radiance of blue light is required here for minimal flicker than at the eccentric point. The log ratio of the radiance of blue light needed at the fovea (Bfov) compared with that needed at the parafovea (Bref) gives a measure of MPOD, i.e., MPOD $=\log ($ Bfov/Bref $) .{ }^{21}$ It should be noted that the chief (but not only) purpose of the eccentric measure is to cancel out the effect of any blue light absorption by the crystalline lens (which happens as a consequence of age-related lens yellowing) so that the final density value is a representation of MP alone, and not MP plus lens yellowing. ${ }^{27}$

The MPS 9000 (also known as M|POD or QuantifEye; Tinsley Ophthalmic, Surrey, UK - referred to as the MPS from now on) adopts a novel approach to measurement of MPOD by HFP. Instead of responding to minimal or no flicker, subjects respond to the appearance of flicker as the blue-green alternation rate is automatically decreased in steps of $6 \mathrm{~Hz}$ from a starting level of $60 \mathrm{~Hz} .^{28}$ This is above the critical flicker fusion frequency for the test conditions and therefore subjects do not perceive any flicker initially. Rather than the radiance of one wavelength being adjusted by the subject, a sequence of blue-green ratios is used, and these are inverse-yoked to ensure that overall luminance remains constant. The instrument also offers the possibility of estimating MPOD from a central measure alone, the peripheral measure being estimated from the age of the subject and their expected level of lens yellowing. ${ }^{27}$

We have previously reported that the inter-session coefficient of repeatability (CR) of the MPS, when used in a clinical setting according to manufacturer guidelines, is between 0.28 and $0.33 .^{29}$ We were keen to find out if this could be improved. In this paper we present techniques and data that show how the CR can be reduced to levels that make the instrument more suitable for monitoring MPOD over time.

\section{MATERIALS AND METHOdS}

\section{Subjects}


For an initial intra-session repeatability study, 25 healthy participants ( 4 males, 21 females) were recruited from staff and students of Aston University, with an age range of 20 to 50 years (mean \pm SD: $29.4 \pm 6.9$ years). Refractive error (best vision sphere, BVS) was between $+1.00 \mathrm{DS}$ and $-10.50 \mathrm{DS}$ (mean $\pm \mathrm{SD}$ : $-2.25 \pm 3.25 \mathrm{DS}$ - all eyes averaged). Visual acuity (VA) was measured under standard testing conditions using a logMAR chart; all eyes had VAs of 0.06 logMAR (Snellen $\left.6 / 7.5^{+2}\right)$ or better. Nine of the subjects had prior experience with the MPS.

Following the intra-session study, a separate group of 27 healthy participants (11 males, 16 females) took part in an inter-session repeatability study. Their ages ranged from 19 to 52 years (mean $\pm S D: 29.9 \pm 10.1$ years), BVS was between +3.00 DS and -8.75 DS (mean $\pm S D:-1.50 \pm 3.00$ DS) in the eye being tested (the right eye for all but one subject), and VA was 0.14 logMAR (Snellen $6 / 7.5^{-2}$ ) or better. Thirteen of these subjects had prior experience with the MPS.

Aston University's Ethics Committee approved the studies. All subjects signed an informed consent form, and all procedures adhered to the tenets of the Declaration of Helsinki.

\section{Data Collection}

The MPS is a small, portable device that has been described in detail elsewhere. ${ }^{28}$ In brief, for the foveal ('central') test, subjects look directly at a central target composed of blue and green lightemitting diodes (LEDs). The alternation rate of the blue and green LEDs is decreased until the subject presses a response button to register their first awareness of the stimulus flickering. This plots a point on a graph that is visible to the operator via a linked computer screen. The process then starts again. The first five responses are used to establish the flicker sensitivity of the subject so that the test can then be conducted at the flicker frequency best suited to each individual subject. Based on this, the main part of the test begins automatically, with the subject responding to their first perception of flicker throughout a series of green-blue ratios, until a V-shaped curve is plotted on the screen (figure 1) - the minimum point on the curve corresponds to equiluminance of the blue and green lights. ${ }^{28}$ The process is repeated for the perifoveal ('peripheral') test where the subject's gaze is directed to a red target that is eight degrees eccentric from the centre, but the 
task is still to respond to their first awareness of flicker in the central stimulus. An internal algorithm calculates the MPOD using the difference between the central and peripheral minima (the larger the difference, the higher the MPOD), although this can also be calculated manually if necessary.

Figure 1 here

In the current investigations, all subjects were tested by the same investigator $(\mathrm{OH})$. They were directed to rest their forehead on the upper part of the instrument's eyepiece, such that the eye being tested was comfortably centered on the appropriate target. An explanation of how to perform the MPS test was given verbally, using an instruction sheet. Subjects wore their habitual distance spectacles/contact lenses, if appropriate, and the eye not being measured was occluded to avoid distraction. The test room was occupied only by the operator and subject, providing a quiet, calm environment.

For the intra-session repeatability study, each participant had their right eye (RE) and left eye (LE) assessed alternately, with three repeats per eye, i.e., R1, L1, R2, L2, R3, L3. The RE was always tested first, and before the central and peripheral tests of R1 and L1, a short practice test was conducted (the practice option is part of the MPS software). The total procedure time, including regular, short breaks to help avoid fatigue, was approximately 45 minutes.

For the inter-session repeatability study, after the short central and peripheral practices, each participant completed between two and three central and peripheral tests at each visit (visits were 7-14 days apart), according to the protocol described later. The total procedure time per visit was between 10 and 20 minutes.

\section{Post-Data-Collection Secondary Analysis}

Because the MPS generates a visible curve as subjects respond to flicker, it provides a way for the operator to assess whether the curve adheres to the expected $\mathrm{V}$-shape, and hence gives an indication as to the accuracy of the MPOD value produced. Many of the curves generated by 
participants in the intra-session study did not conform to the ideal V-shape with a clearly-defined minimum, but they still produced an MPOD value and were considered acceptable by us at the time, based on knowledge acquired from the product manual ('MPOD QuantifEye Reference Guide and Technician Training'). We assessed the influence on repeatability of data from these less than optimal curves. Hence, further careful observation of all subjects' curves took place; MPOD values generated from curves without a clear-cut minimum area were removed, and MPOD values generated from curves with questionable minima were adjusted (see figure 2 and table 1). It should be noted that the product literature advises that MPOD values from graphs that are "scattered significantly" should not be relied upon as accurate, but the visual examples of unacceptable graphs are far removed from anything resembling a V-curve, and therefore it was difficult for us to determine what the cut-off point for significant scatter was, particularly as very few subjects exhibited consistently 'perfect' curves. During the data collection, the operator did discard and repeat any tests that were judged (subjectively) to be of unacceptable quality at the time.

Figure 2 here

Table 1 here

\section{Statistics}

Microsoft Excel and IBM SPSS were used for data analysis. Simple calculations were used to derive mean individual standard deviations. Test-retest repeatability (intra- and inter-session) was determined using Bland-Altman analysis. ${ }^{30}$ The differences between the intra-session CRs before and after data removal/adjustment were assessed by one-way repeated measures ANOVA (each data set was normally distributed).

Accurate analysis of test-retest data can be achieved using the $\mathrm{CR},{ }^{30}$ which gives the $95 \%$ confidence limits for the difference between two sets of results. It is calculated as 1.96 multiplied by the standard deviation of the differences between two sets of data, and indicates the amount of change that can occur between readings and still be classed as measurement noise. In other 
words, only increases or decreases in MPOD greater than the CR should be classed as clinically detectable.

For the intra-session data, a one-way repeated measures ANOVA was conducted to analyse the original $C R$ values $(O)$, the $C R$ values after data removal $(R)$, and the $C R$ values after data removal and adjustment (RA). Mauchly's test indicated that the assumption of sphericity was not met, and as a result, degrees of freedom were corrected using the Greenhouse-Geisser estimate of sphericity.

\section{RESULTS}

Table 2 here

The intra-session mean individual standard deviations of the MPOD values are given in table 3 for each test condition. In other words - taking 'CP RE' as an example - the standard deviation of each individual's three CP (central and peripheral testing) RE MPOD values was worked out, followed by calculation of the mean of all 25 standard deviations. After adjustment of MPOD values as appropriate (minima adjustment), the standard deviations were slightly lower in all categories. Note that three MPOD values were obtained for each test condition, and no data was removed for this particular analysis.

Table 3

The intra-session CR values for MPOD, before and after data removal and minima adjustment, are shown in table 4 . There was a statistically significant improvement in repeatability $(F(1.2,8.4)=$ 11.09, $p=0.008$ ). Pairwise comparisons (Bonferroni corrected) indicated a statistically significant difference between $O$ and RA $(p<0.05)$, and between R and RA $(p<0.05)$, but not between $O$ and R $(p>0.05)$. Figures 3 and 4 illustrate in graphical format (Bland-Altman plots) the repeatability for the various data sets. 
Table 4 here

Figure 3 here

Figure 4 here

Table 5 here

The original raw intra-session MPOD difference data was also considered. It was calculated that with the CP MPOD data, between $64 \%$ (R1 vs R2) and 80\% (L2 vs L3) of participants' MPOD values were within 0.09 of each other from one test to the next. With the C-only (central testing only, age-based peripheral estimate provided by the MPS) MPOD data, between $84 \%$ (R1 vs R2) and $92 \%$ (R2 vs R3, L1 vs L2, and L2 vs L3) were within 0.09 of each other from one test to the next. These reasonably high percentages imply that our CR values may be influenced by individuals who simply struggle to perform the flicker test well. Indeed, taking into account the removed MPOD values, plus the adjusted values, the above percentages increased to $80-91 \%$ and 86-100\%, for CP MPOD and C-only MPOD respectively. To explore this further, table 5 is a subject-specific breakdown of where and how much data was removed. It shows that few subjects had more than one central minimum value or one peripheral minimum value removed, thus indicating that most subjects achieved at least two optimal central and peripheral curves per eye (albeit with some later adjustments), and this was seemingly irrespective of whether subjects had some previous experience of using the MPS or not. One subject, in particular, clearly struggled with the peripheral MPS task, resulting in the removal of five of their six peripheral curves.

An MPOD reading that is repeatable to within 0.09 seems like an acceptable limit of accuracy for a psychophysical task of this nature. Combined with our other findings, this led to the following protocol suggestion:

- For reliable results, each subject should perform the central and peripheral test twice, even if the first curves are considered to be 'perfect'. 
- If there is $\mathrm{a} \geq 0.4 \mathrm{~dB}$ difference between equivalent minimum readings (central or peripheral), or if one or more of the generated curves are less than optimal in appearance (and not amenable to adjustment), then a third central and/or peripheral test (as appropriate) should be completed.

- When a curve exhibits a poorly-defined minimum, it should not be included in the final calculation of MPOD, but adjustment of a computer-produced minimum reading is acceptable when a curve has two or three very similar minimum points.

This method will lead to most subjects having at least two good-quality central and peripheral curves, and from these the accepted minima can be averaged, followed by calculation of MPOD using the earlier-established table (table 1). The reason for choosing a difference of $0.4 \mathrm{~dB}$ as the cut-off point at which a repeat test should be completed is because this equates to a 0.09 or 0.1 difference in MPOD (see table 1). It could have been decided to use $a \geq 0.1$ difference in MPOD readings as the point at which to repeat a test. The problem with this, however, is that it requires both tests to be repeated (central and peripheral), which may be unnecessary. By concentrating on the individual curves (and their minimum values), the testing time can be reduced.

The protocol above was subsequently tested in terms of inter-session repeatability with our second group of subjects. Using this technique, all subjects had two to three good quality central and peripheral curves to derive MPOD from, and no subjects needed to be removed from the analysis. The mean CP MPOD for the 27 participants in the inter-session study was $0.35 \pm 0.16$ at visits one and two. The mean C-only MPOD was $0.38 \pm 0.18$ and $0.39 \pm 0.18$ at visits one and two respectively. Table 6 and figure 5 show the inter-session agreement between MPOD values. The CRs were 0.084 and 0.063 for CP and C-only MPOD respectively.

Table 6 here

Figure 5 here 


\section{Discussion}

Through data interpretation and procedural changes, we aimed to improve the repeatability of the MPS, and assessed the validity of doing so by means of various statistical indicators.

In a study by van der Veen et al., ${ }^{28} 26$ subjects repeated MPS measurements (CP) five times in a single session, and the mean individual standard deviation was $0.067 \pm 0.033$. The results of the present investigation are not directly comparable because there were only three repeats per eye, and the same eye was not tested consecutively. Nevertheless, the figures are similar, with our CP RE mean individual standard deviation being $0.063 \pm 0.047$. In all four test conditions, minima adjustment alone successfully improved (i.e., reduced) the mean individual standard deviation of the three repeated MPOD measurements. This was encouraging, as it demonstrated that the adjustment technique is beneficial.

A statistically significant improvement in the intra-session repeatability was obtained through removal and adjustment of the data. The weakest CR was between the first and second CP RE MPOD tests $(0.25)$. The CR was reduced to 0.16 following removal of less than optimal data, although this involved a fairly high number of such removals (11 out of 50 MPOD values). However, we also found that the majority of our participants produced serial MPOD values within 0.09 of each other, and moreover that most subjects achieved at least two (out of three) optimal central and peripheral curves per eye (with some minima adjustments). This led to a protocol suggestion that aimed to improve on previously-reported MPS-based MPOD CR values. The protocol was assessed using 27 volunteers, and the result was an inter-session (CP MPOD) CR of 0.084. This is a considerable improvement on the CRs of $0.31(n=40), 0.18(n=20)$ and 0.19 $(n=25)$, as reported by Bartlett et al., ${ }^{29}$ de Kinkelder et al.$^{31}(2011)$ and Loughman et al. ${ }^{32}$ respectively. The ages of the participants in these studies were very similar to the current one. This new CR is also as good as, if not better than, any stated HFP reliability of the last 15 years. ${ }^{33,34}$ It could be argued that the intra-session results were influenced by the fact that about half of the participants were already familiar with the MPS, especially as the mean differences between our first measurements sets (R1 and R2) are larger than the mean differences between our last 
measurement sets (L2 and L3) However, further analysis of the inter-session data, using only those with no prior experience on the MPS $(n=14)$, resulted in a CR of 0.072 and 0.043 for C-only MPOD. It is also important to bear in mind that intervention studies have demonstrated only small increases in MPOD (rarely more than 0.07 ) with lutein/zeaxanthin supplementation ${ }^{33}$ and so HFP instruments may not be able to detect these changes.

A high level of inter-session repeatability is important if MPOD is to be accurately monitored over time, especially in an intervention scenario where an individual is attempting to increase their L/Z/MZ intake via dietary modification and/or supplement use. Therefore, we recommend following the protocol that we have described when using the MPS to monitor changes in MPOD.

A further point to consider on this matter is the C-only MPOD repeatability. For the purposes of monitoring MPOD over time, the central test alone could suffice, because the emphasis is on whether there is any change in MPOD, rather than whether the MPOD value is an accurate representation of the subject's actual MP. ${ }^{27}$ To this end, we observed in our intra-session study that between $80 \%$ (R1 vs R2) and 92\% (L2 vs L3) of participants' MPOD values were within 0.05 of each other from one test to the next. Furthermore, the removal of MPOD values generated from curves with poorly-defined minima, plus our adjusted values, increased these percentages to between $86 \%$ and $96 \%$. Consequently, it may be easier to detect a clinically significant change in an individual's MPOD by using the C-only MPOD value. This is supported by the inter-session CR of 0.063 , as reported above.

Despite the improved repeatability demonstrated using the protocol, we concede that in a clinical setting, it may be considered too convoluted, and the calculations themselves too time-consuming. It might therefore be more appropriate to use a slightly different protocol, albeit resulting in one or two unnecessary tests: Each patient could perform the central and peripheral test twice, even if the first curves are considered to be 'perfect'. If there is $a \geq 0.1$ difference between MPOD readings, or if one or more of the generated curves are less than optimal in appearance (and not amenable to adjustment), then a third central and peripheral test should be completed. The average of the two 
or three MPOD values should give an accurate measure of MP, unless many of the curves are poor or the MPOD values constantly variable, in which case it would be worth paying attention to the central curves and C-only MPOD readings to see whether the results are more consistent. It could be argued that the examiner still has a great influence on the final value measured, as he or she has to make a subjective decision on the quality of a measurement. One way to get around this problem would be to alter the software such that all inadequate measurements are disregarded, and a repeat test is forced when the difference between successive measurements exceeds $0.4 \mathrm{~dB}$.

In summary, we propose an evidence-based testing protocol that improves the repeatability of the MPS, making the instrument more useful in clinical and research environments.

\section{REFERENCES}

1. Bone RA, Landrum JT, Hime GW, Cains A, Zamor J (1993) Stereochemistry of the human macular carotenoids. Invest Ophthalmol Vis Sci 34:2033-2040.

2. Malinow MR, Feeney-Burns L, Peterson LH, Klein ML, Neuringer M (1980) Diet-related macular anomalies in monkeys. Invest Ophthalmol Vis Sci 19:857-863.

3. Neuringer M, Sandstrom MM, Johnson EJ, Snodderly DM (2004) Nutritional manipulation of primate retinas, I: effects of lutein or zeaxanthin supplements on serum and macular pigment in xanthophyll-free rhesus monkeys. Invest Ophthalmol Vis Sci 45:3234-3243.

4. Hammond BR, Johnson EJ, Russell RM, Krinsky NI, Yeum KJ, Edwards RB, Snodderly DM (1997) Dietary modification of human macular pigment density. Invest Ophthalmol Vis Sci 38:17951801.

5. Wenzel AJ, Gerweck C, Barbato D, Nicolosi RJ, Handelman GJ, Curran-Celentano J (2006) A 12-wk egg intervention increases serum zeaxanthin and macular pigment optical density in women. J Nutr 136:2568-2573. 
6. Connolly EE, Beatty S, Thurnham DI, Loughman J, Howard AN, Stack J, Nolan JM (2010) Augmentation of macular pigment following supplementation with all three macular carotenoids: an exploratory study. Curr Eye Res 35:335-351.

7. Wyszecki G, Stiles WS (1982) The eye. In: Color Science: Concepts and Methods, Quantitative Data and Formulae. Wiley-Interscience, New York, pp 112-114.

8. Snodderly DM, Brown PK, Delori FC, Auran JD (1984) The macular pigment. I. Absorbance spectra, localization, and discrimination from other yellow pigments in primate retinas. Invest Ophthalmol Vis Sci 25:660-673.

9. Handelman GJ, Snodderly DM, Krinsky NI, Russett MD, Adler AJ (1991) Biological control of primate macular pigment - biochemical and densitometric studies. Invest Ophthalmol Vis Sci $32: 257-267$.

10. Bone RA, Landrum JT, Cains A (1992) Optical density spectra of the macular pigment in vivo and in vitro. Vision Res 32:105-110.

11. Hammond BR, Caruso-Avery M (2000) Macular pigment optical density in a southwestern sample. Invest Ophthalmol Vis Sci 41:1492-1497.

12. Landrum JT, Bone RA (2001) Lutein, zeaxanthin, and the macular pigment. Arch Biochem Biophys 385:28-40.

13. Chucair AJ, Rotstein NP, SanGiovanni JP, During A, Chew EY, Politi LE (2007) Lutein and zeaxanthin protect photoreceptors from apoptosis induced by oxidative stress: relation with docosahexaenoic acid. Invest Ophthalmol Vis Sci 48:5168-5177. 
14. Khachik F, Bernstein PS, Garland DL (1997) Identification of lutein and zeaxanthin oxidation products in human and monkey retinas. Invest Ophthalmol Vis Sci 38:1802-1811.

15. Beatty S, Koh HH, Henson D, Boulton M (2000) The role of oxidative stress in the pathogenesis of age-related macular degeneration. Surv Ophthalmol 45:115-134.

16. Algvere PV, Marshall J, Seregard S (2006) Age-related maculopathy and the impact of blue light hazard. Acta Ophthalmol Scand 84:4-15.

17. Beatty S, Murray IJ, Henson DB, Carden D, Koh HH, Boulton ME (2001) Macular pigment and risk for age-related macular degeneration in subjects from a Northern European population. Invest Ophthalmol Vis Sci 42:439-446.

18. Bone RA, Landrum JT, Mayne ST, Gomez CM, Tibor SE, Twaroska EE (2001) Macular pigment in donor eyes with and without AMD: A case-control study. Invest Ophthalmol Vis Sci 42:235-240.

19. Richer S, Stiles W, Statkute L, Pulido J, Frankowski J, Rudy D, Pei K, Tsipursky M, Nyland J (2004) Double-masked, placebo-controlled, randomized trial of lutein and antioxidant supplementation in the intervention of atrophic age-related macular degeneration: the Veterans LAST study (Lutein Antioxidant Supplementation Trial). Optometry 75:216-230

20. Nolan JM, Stack J, O'Donovan O, Loane E, Beatty S (2007) Risk factors for age-related maculopathy are associated with a relative lack of macular pigment. Exp Eye Res 84:61-74.

21. Snodderly DM, Hammond BR (1999) In vivo psychophysical assessment of nutritional and environmental influences on human ocular tissues: lens and macular pigment. In: Taylor A (ed) Nutritional and Environmental Influences on the Eye. Boca Raton FL, CRC Press, pp 251-273. 
22. Loane E, Stack J, Beatty S, Nolan JM (2007) Measurement of macular pigment optical density using two different heterochromatic flicker photometers. Curr Eye Res 32:555-564.

23. Kirby ML, Galea M, Loane E, Stack J, Beatty S, Nolan JM (2009) Foveal anatomic associations with the secondary peak and the slope of the macular pigment spatial profile. Invest Ophthalmol Vis Sci 50:1383-1391.

24. Hammond BR, Wooten BR, Snodderly DM (1997) Individual variations in the spatial profile of human macular pigment. J Opt Soc Am A Opt Image Sci Vis 14:1187-1196.

25. Landrum JT, Bone RA, Joa H, Kilburn MD, Moore LL, Sprague KE (1997) A one year study of the macular pigment: the effect of 140 days of a lutein supplement. Exp Eye Res 65:57-62.

26. Wooten BR, Hammond BR, Land RI, Snodderly DM (1999) A practical method for measuring macular pigment optical density. Invest Ophthalmol Vis Sci 40:2481-2489.

27. Makridaki M, Carden D, Murray IJ (2009) Macular pigment measurement in clinics: controlling the effect of the ageing media. Ophthalmic Physiol Opt 29:338-344.

28. van der Veen RLP, Berendschot TTJM, Hendrikse F, Carden D, Makridaki M, Murray IJ (2009) A new desktop instrument for measuring macular pigment optical density based on a novel technique for setting flicker thresholds. Ophthalmic Physiol Opt 29:127-137.

29. Bartlett H, Stainer L, Singh S, Eperjesi F, Howells O (2010) Clinical evaluation of the MPS 9000 Macular Pigment Screener. Br J Ophthalmol 94:753-756.

30. Bland JM, Altman DG (1986) Statistical methods for assessing agreement between two methods of clinical measurement. Lancet 1:307-310. 
31. de Kinkelder R, van der Veen RLP, Verbaak FD, Faber DJ, van Leeuwen TG, Berendschot TTJM (2011) Macular pigment optical density measurements: evaluation of a device using heterochromatic flicker photometry. Eye 25:105-112.

32. Loughman J, Scanlon G, Nolan JM, O'Dwyer V, Beatty S (2012) An evaluation of a novel instrument for measuring macular pigment optical density: the MPS 9000. Acta Ophthalmol 90:e90-e97.

33. Bartlett $H$, Howells O, Eperjesi $F(2010)$ The role of macular pigment assessment in clinical practice: a review. Clin Exp Optom 93:300-308.

34. Howells O, Eperjesi F, Bartlett $H$ (2011) Measuring macular pigment optical density in vivo: a review of techniques. Graefes Arch Clin Exp Ophthalmol 249:315-347.

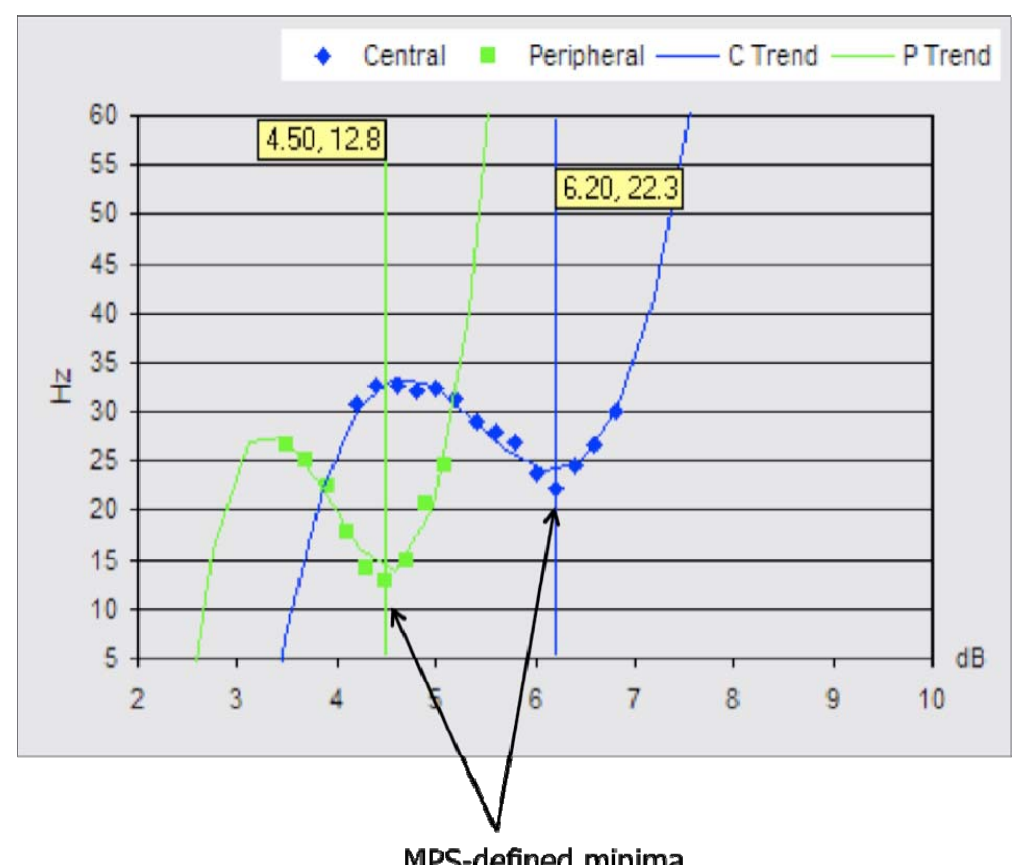

Figure 1. Example of an optimal central curve and an optimal peripheral curve - both V-shaped with well-defined minima. 


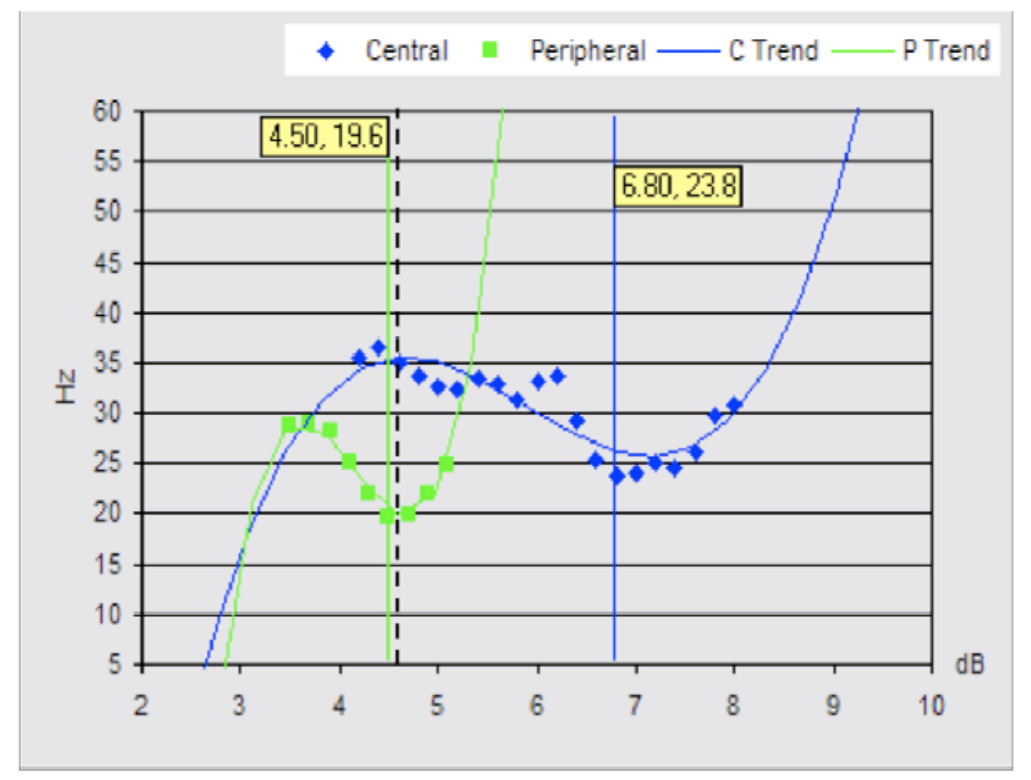

Figure 2. Example of a less than optimal central curve (diamond points - right curve) and a peripheral curve (square points - left curve) with two possible minima.

The central curve is less than optimal because it has several points all with similar flicker frequencies to the computer-chosen exact minimum at $6.80 \mathrm{~dB}$, rather than an isolated, definite minimum. We removed an MPOD value generated by a curve such as this from our repeatability analysis. The peripheral curve has an exact minimum at $4.50 \mathrm{~dB}$ but, judging by eye, its right adjacent point has a very similar flicker frequency that could also be argued to be a minimum (at $4.70 \mathrm{~dB}$ ). We would adjust an MPOD value generated by a curve such as this, by recalculation using a peripheral minimum of $4.60 \mathrm{~dB}$, i.e., the mean of these two points (the dashed line indicates the adjusted minimum) 


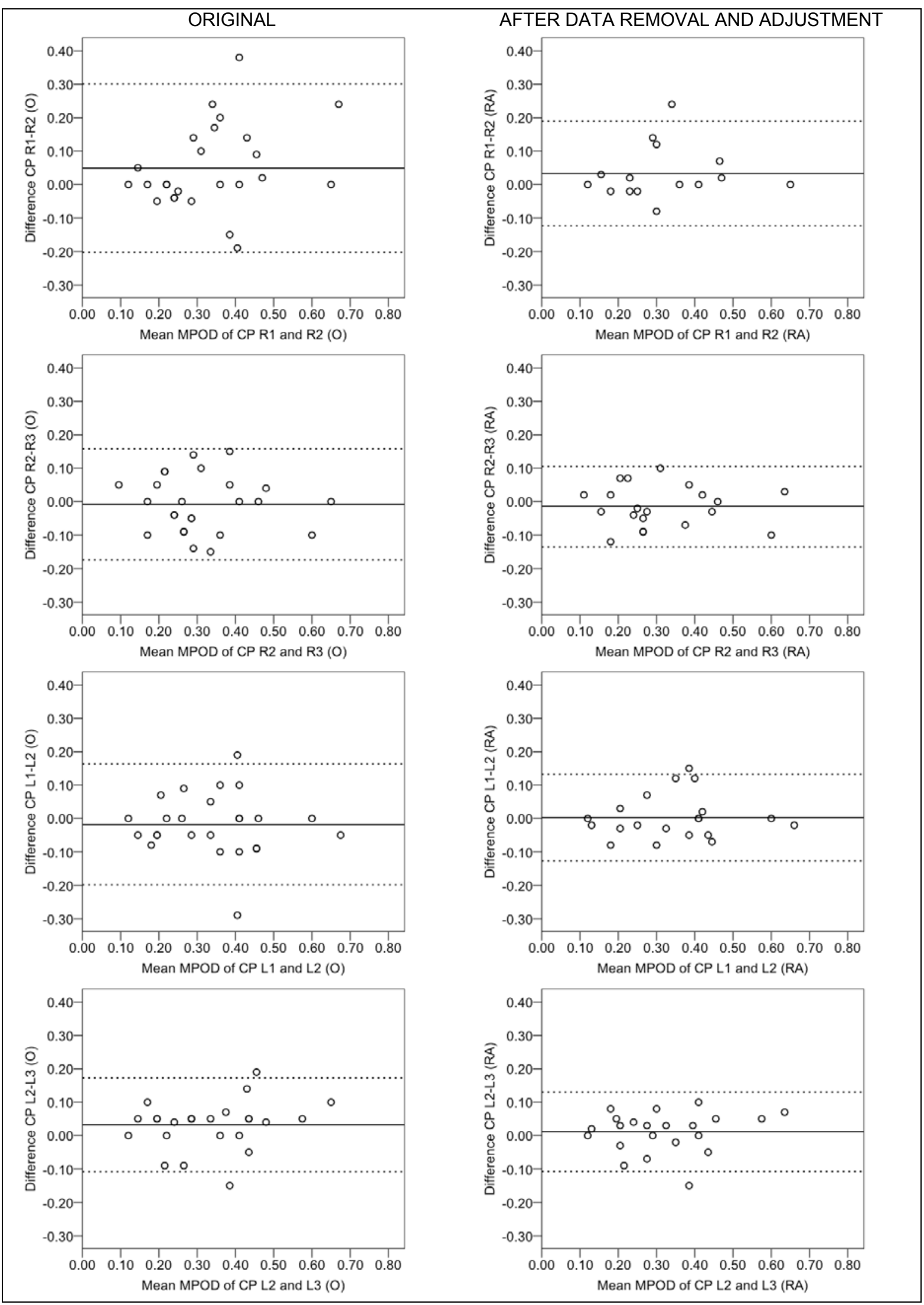

Figure 3. Bland-Altman plots representing the difference in CP testing derived MPOD readings between measures, compared with the mean of both measures. The solid line represents the mean difference, and the dashed lines represent the $95 \%$ confidence limits. 
Left plots $=$ original data $(O)$, i.e., before removal and adjustment. Right plots $=$ after data removal and minima adjustment (RA).

NB There are several overlapping points in each plot 


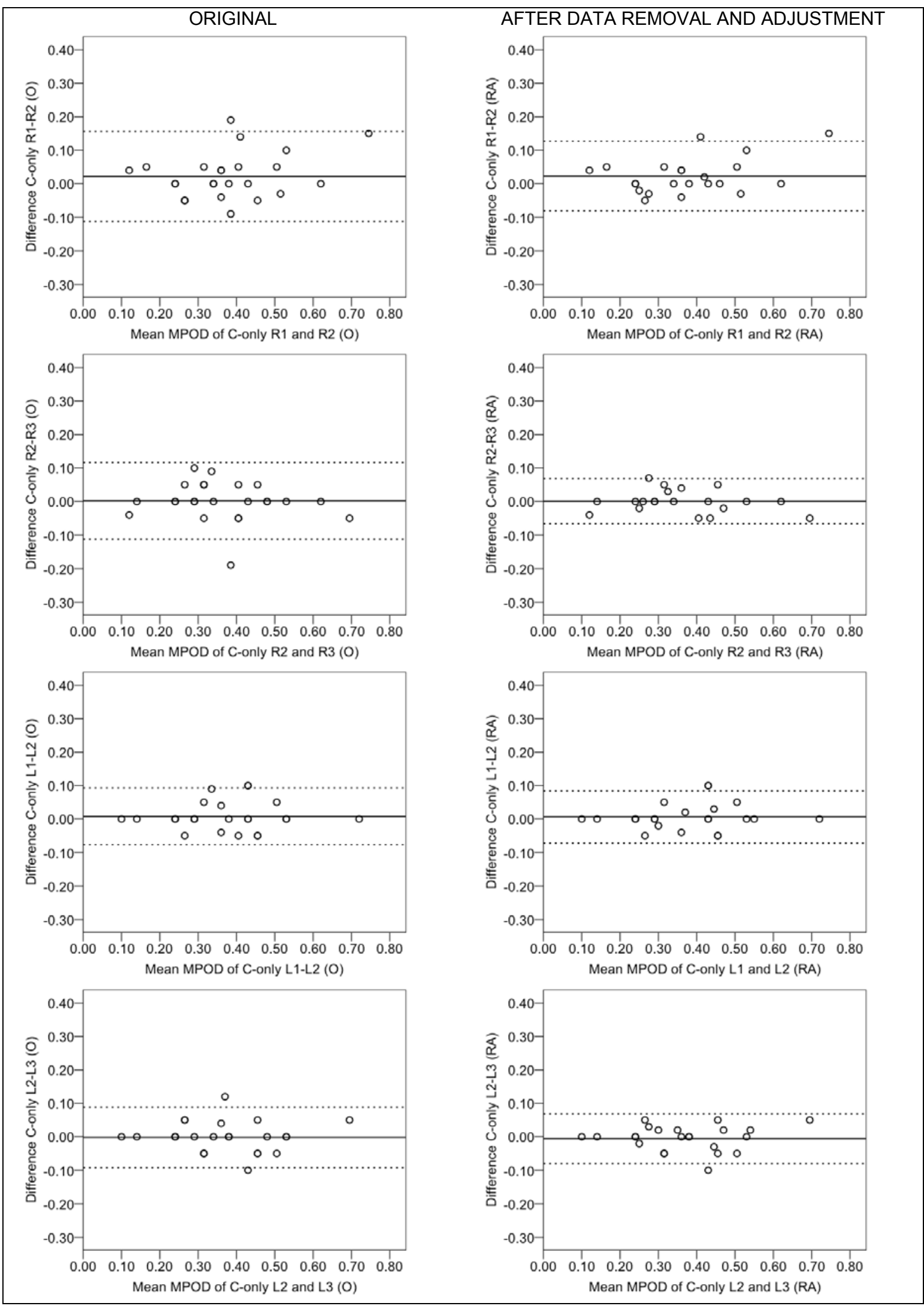

Figure 4. Bland-Altman plots representing the difference in C-only derived MPOD readings between measures, compared with the mean of both measures. The solid line represents the mean difference, and the dashed lines represent the 95\% confidence limits.

NB There are several overlapping points in each plot. 

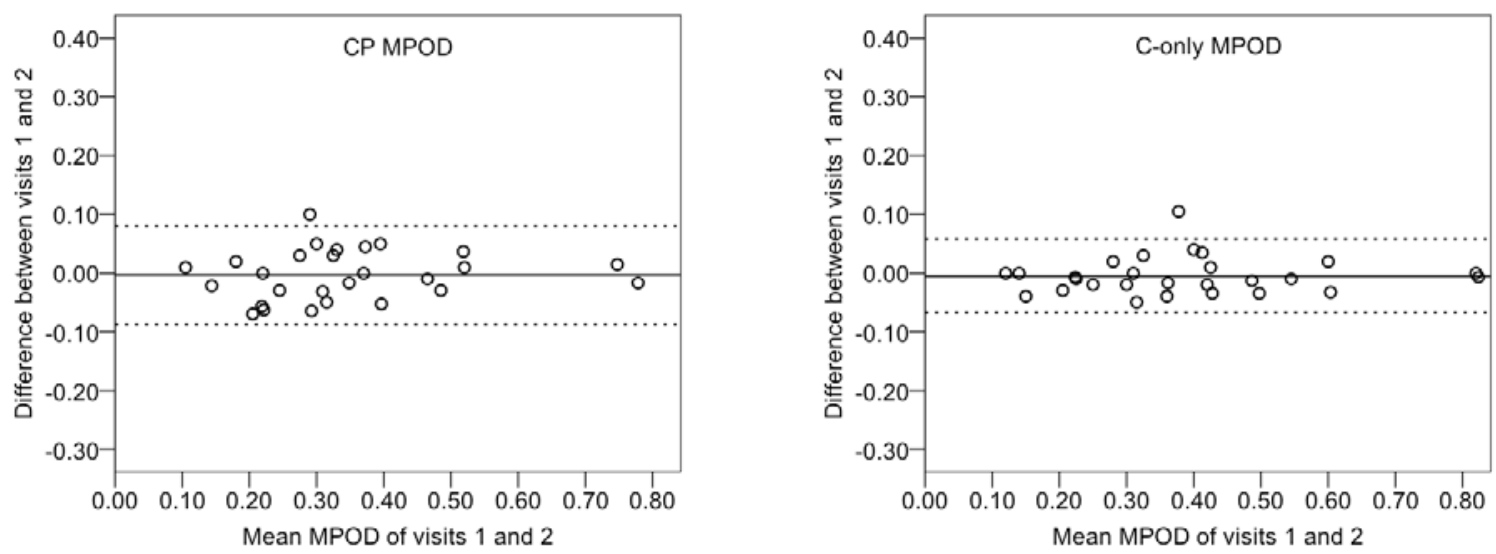

Figure 5. Bland-Altman plots representing the difference in MPOD readings between two visits, compared with the mean of both visits. The solid line represents the mean difference, and the dashed lines represent the $95 \%$ confidence limits.

\begin{tabular}{|c|c|c|c|}
\hline $\begin{array}{c}\text { Difference: } \\
\text { C min - P min }\end{array}$ & MPOD & $\begin{array}{c}\text { Difference: } \\
\text { C min - P min }\end{array}$ & MPOD \\
\hline 0.1 & 0.02 & 2.1 & 0.5 \\
\hline 0.2 & 0.05 & 2.2 & 0.53 \\
\hline 0.3 & 0.07 & 2.3 & 0.55 \\
\hline 0.4 & 0.1 & 2.4 & 0.58 \\
\hline 0.5 & 0.12 & 2.5 & 0.6 \\
\hline 0.6 & 0.14 & 2.6 & 0.62 \\
\hline 0.7 & 0.17 & 2.7 & 0.65 \\
\hline 0.8 & 0.19 & 2.8 & 0.67 \\
\hline 0.9 & 0.22 & 2.9 & 0.7 \\
\hline 1.0 & 0.24 & 3.0 & 0.72 \\
\hline 1.1 & 0.26 & 3.1 & 0.74 \\
\hline 1.2 & 0.29 & 3.2 & 0.77 \\
\hline 1.3 & 0.31 & 3.3 & 0.79 \\
\hline 1.4 & 0.34 & 3.4 & 0.82 \\
\hline 1.5 & 0.36 & 3.5 & 0.84 \\
\hline 1.6 & 0.38 & 3.6 & 0.86 \\
\hline 1.7 & 0.41 & 3.7 & 0.89 \\
\hline 1.8 & 0.43 & 3.8 & 0.91 \\
\hline 1.9 & 0.46 & 3.9 & 0.94 \\
\hline 2.0 & 0.48 & 4.0 & 0.96 \\
\hline
\end{tabular}

Table 1. Conversion table for MPS-based MPOD.

New MPOD values, following adjustment (by eye) of minimum points on the curves, were calculated as follows:

Difference between central minimum $(\mathrm{C}$ min) and peripheral minimum $(\mathrm{P} \min )=X$

$X=M P O D$, found by referring to the table. Example: $C \min =6.1 \mathrm{~dB}, P \min =4.7 \mathrm{~dB} ; 6.1-4.7=$ $1.4 ; 1.4=$ MPOD value of 0.34 .

The authors produced this conversion table after much practice on the MPS. 


\begin{tabular}{|l|c|c|c|}
\hline \multirow{2}{*}{ Test condition } & \multicolumn{3}{|c|}{ Mean ( \pm SD) MPOD } \\
\cline { 2 - 4 } & Measure 1 & Measure 2 & Measure 3 \\
\hline CP RE & $0.36 \pm 0.17$ & $0.31 \pm 0.13$ & $0.32 \pm 0.14$ \\
\hline CP LE & $0.33 \pm 0.14$ & $0.35 \pm 0.15$ & $0.32 \pm 0.13$ \\
\hline C-only RE & $0.39 \pm 0.15$ & $0.37 \pm 0.13$ & $0.36 \pm 0.14$ \\
\hline C-only LE & $0.37 \pm 0.14$ & $0.36 \pm 0.13$ & $0.36 \pm 0.14$ \\
\hline
\end{tabular}

Table 2. The mean MPODs of the 25 subjects participating in the intra-session study.

$\mathrm{CP}=$ central and peripheral testing derived MPOD. C-only = central testing only derived MPOD (peripheral estimate provided by the MPS, based on subject age). RE = right eye. LE = left eye.

\begin{tabular}{|c|c|c|}
\hline Test condition & $\begin{array}{c}\text { Mean SD between } \\
\text { measures 1, 2 \& 3 }\end{array}$ & $\begin{array}{c}\text { Mean SD after } \\
\text { minima adjustment }\end{array}$ \\
\hline CP RE & $0.063 \pm 0.047$ & $0.060 \pm 0.045$ \\
\hline CP LE & $0.047 \pm 0.030$ & $0.041 \pm 0.028$ \\
\hline C-only RE & $0.038 \pm 0.027$ & $0.034 \pm 0.027$ \\
\hline C-only LE & $0.024 \pm 0.021$ & $0.023 \pm 0.019$ \\
\hline
\end{tabular}

Table 3. The means of the individual standard deviations of three repeated MPOD measurements in 25 subjects, demonstrated for each of the four test conditions, before and after minima adjustment.

\begin{tabular}{|c|c|c|c|c|c|}
\hline \multirow[b]{2}{*}{ Comparison } & \multicolumn{3}{|c|}{ Coefficient of repeatability } & \multirow[b]{2}{*}{$\begin{array}{c}\text { Number of } \\
\text { MPOD values } \\
\text { from a total of } 50 \\
\text { removed }\end{array}$} & \multirow[b]{2}{*}{$\begin{array}{l}\text { Remainder of } \\
\text { MPOD values } \\
\text { with minima } \\
\text { adjusted }\end{array}$} \\
\hline & Original $(\mathrm{O})$ & $\begin{array}{c}\text { After data } \\
\text { removal }(R)\end{array}$ & $\begin{array}{c}\text { After data } \\
\text { removal \& } \\
\text { adjustment of } \\
\text { minima (RA) }\end{array}$ & & \\
\hline CP R1, R2 & 0.25 & 0.16 & 0.16 & $11(n=10)$ & $7(n=7)$ \\
\hline CP R2, R3 & 0.17 & 0.15 & 0.12 & $7(n=5)$ & $17(n=15)$ \\
\hline CP L1, L2 & 0.18 & 0.15 & 0.13 & $6(n=5)$ & $18(n=16)$ \\
\hline CP L2, L3 & 0.14 & 0.13 & 0.12 & $4(n=3)$ & $18(n=16)$ \\
\hline C-only R1, R2 & 0.13 & 0.11 & 0.10 & $3(n=3)$ & $5(n=4)$ \\
\hline C-only R2, R3 & 0.11 & 0.08 & 0.07 & $5(n=5)$ & $9(n=7)$ \\
\hline C-only L1, L2 & 0.08 & 0.08 & 0.08 & $2(n=2)$ & $6(n=5)$ \\
\hline C-only L2, L3 & 0.09 & 0.08 & 0.07 & $1(n=1)$ & $9(n=8)$ \\
\hline
\end{tabular}

Table 4. Intra-session coefficients of repeatability for the various data sets, before and after data removal and minima adjustment. $\mathrm{R} 1, \mathrm{~L} 2$, etc. = right eye measure 1 , left eye measure 2 , etc. $n=$ number of subjects (out of 25). 


\begin{tabular}{|c|c|c|c|c|c|c|c|c|}
\hline \multicolumn{2}{|c|}{ Number of minimum values removed: } & $\mathbf{0}$ & 1 & 2 & 3 & 4 & 5 & 6 \\
\hline \multirow{3}{*}{ 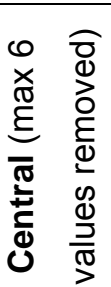 } & Total number of subjects & 18 & 5 & 2 & 0 & 0 & 0 & 0 \\
\hline & $\begin{array}{l}\text { Number of subjects with previous } \\
\text { experience }\end{array}$ & $\begin{array}{c}7 \\
(78)\end{array}$ & $\begin{array}{c}1 \\
(11)\end{array}$ & $\begin{array}{c}1 \\
(11)\end{array}$ & $\begin{array}{c}0 \\
(0)\end{array}$ & (0) & $\begin{array}{c}0 \\
(0)\end{array}$ & $\begin{array}{c}0 \\
(0)\end{array}$ \\
\hline & $\begin{array}{l}\text { Number of subjects with NO } \\
\text { previous experience }\end{array}$ & $\begin{array}{c}11 \\
(69)\end{array}$ & $\begin{array}{c}4 \\
(25)\end{array}$ & $\begin{array}{c}1 \\
(6)\end{array}$ & $\begin{array}{l}0 \\
(0)\end{array}$ & (0) & $\begin{array}{c}0 \\
(0)\end{array}$ & $\begin{array}{c}0 \\
(0)\end{array}$ \\
\hline \multirow{3}{*}{ 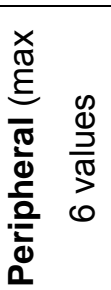 } & Total number of subjects & 15 & 7 & 2 & 0 & 0 & 1 & 0 \\
\hline & $\begin{array}{l}\text { Number of subjects with previous } \\
\text { experience }\end{array}$ & $\begin{array}{c}4 \\
(44)\end{array}$ & $\begin{array}{c}3 \\
(33)\end{array}$ & $\begin{array}{c}2 \\
(22)\end{array}$ & $\begin{array}{c}0 \\
(0)\end{array}$ & (0) & (0) & $\begin{array}{c}0 \\
(0)\end{array}$ \\
\hline & $\begin{array}{l}\text { Number of subjects with } \mathrm{NO} \\
\text { previous experience }\end{array}$ & $\begin{array}{c}11 \\
(69)\end{array}$ & $\begin{array}{c}4 \\
(25)\end{array}$ & $\begin{array}{c}0 \\
(0)\end{array}$ & $\begin{array}{c}0 \\
(0)\end{array}$ & (0) & $\begin{array}{c}1 \\
(6)\end{array}$ & (0) \\
\hline \multirow{3}{*}{ 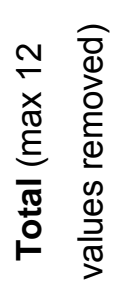 } & Total number of subjects & 12 & 7 & 3 & 2 & 0 & 0 & 1 \\
\hline & $\begin{array}{l}\text { Number of subjects with previous } \\
\text { experience }\end{array}$ & $\begin{array}{c}4 \\
(44)\end{array}$ & $\begin{array}{c}2 \\
(22)\end{array}$ & $\begin{array}{c}1 \\
(11)\end{array}$ & $\begin{array}{c}2 \\
(22)\end{array}$ & (0) & (0) & $\begin{array}{c}0 \\
(0)\end{array}$ \\
\hline & $\begin{array}{l}\text { Number of subjects with } \mathrm{NO} \\
\text { previous experience }\end{array}$ & $\begin{array}{c}8 \\
(50)\end{array}$ & $\begin{array}{c}5 \\
(31)\end{array}$ & $\begin{array}{c}2 \\
(13)\end{array}$ & $\begin{array}{c}0 \\
(0)\end{array}$ & (0) & $\begin{array}{c}0 \\
(0)\end{array}$ & $\begin{array}{c}1 \\
(6)\end{array}$ \\
\hline
\end{tabular}

Table 5. The breakdown of how many minimum values were removed in the secondary analysis, and how many subjects were involved in these removals. Percentages are in brackets.

\begin{tabular}{|l|c|c|}
\cline { 2 - 3 } \multicolumn{1}{c|}{} & CP MPOD1 - MPOD2 & C-only MPOD1 - MPOD2 \\
\hline Mean difference & -0.003 & -0.005 \\
\hline SD of mean difference & 0.043 & 0.032 \\
\hline Coefficient of repeatability & 0.084 & 0.063 \\
\hline
\end{tabular}

Table 6. Determination of inter-session coefficients of repeatability $(n=27)$ using the proposed protocol. 